Yao, J. , Wong, D. W.S., Bailey, N. and Minton, J. (2019) Spatial segregation measures: a methodological review. Tijdschrift voor Economische en Sociale Geografie, 110(3), pp. 235-250.

There may be differences between this version and the published version. You are advised to consult the publisher's version if you wish to cite from it.

This is the peer reviewed version of the following article:

Yao, J. , Wong, D. W.S., Bailey, N. and Minton, J. (2019) Spatial segregation measures: a methodological review. Tijdschrift voor Economische en Sociale Geografie, 110(3), pp. 235-250.

(doi: 10.1111/tesg.12305)

This article may be used for non-commercial purposes in accordance with Wiley Terms and Conditions for Self-Archiving.

$\underline{\text { http://eprints.gla.ac.uk/146758/ }}$

Deposited on: 28 August 2017

Enlighten - Research publications by members of the University of Glasgow http://eprints.gla.ac.uk/ 


\title{
Spatial Segregation Measures: A Methodological Review
}

\author{
Jing Yao ${ }^{1,2}$, David W.S. Wong ${ }^{3}$, Nick Bailey ${ }^{1,2}$, Jon Minton ${ }^{2}$ \\ ${ }^{1}$ Urban Big Data Centre, University of Glasgow, Glasgow, G12 8RZ, UK \\ ${ }^{2}$ School of Social and Political Sciences, University of Glasgow, Glasgow, G12 8RT, UK \\ ${ }^{3}$ Department of Geography and Geolnformation Science, George Mason University, Fairfax, VA 22030, USA
}

Emails: JY: Jing.Yao@glasgow.ac.uk

DW: dwong2@gmu.edu

NB: Nick.Bailey@glasgow.ac.uk

JM: Jonathan.Minton@glasgow.ac.uk 


\title{
Spatial Segregation Measures: A Methodological Review
}

\begin{abstract}
Quantitative indices of segregation are powerful tools for summarising the spatial relationships between population groups and thereby providing the basis for analysis and public policy intervention. While the broad concept of segregation may be intuitive, measurement is challenging because of the complexity of varied dimensions and spatial arrangements. Many traditional measures can be criticised for over-simplification or over-reduction, not least in their treatment of geographical space. Over the last several decades, however, a series of measures has been developed to explicitly incorporate the spatial arrangement of population groups as well as their interactions. This paper reviews the development of spatial segregation measures, particularly focusing on the mathematical formulation of spatial arrangement/relations. In addition, several related issues are discussed, including representation of spatial interaction, spatial scale and statistical inferences. Also, this paper presents an overview of existing software tools that are readily available for calculating some of the reviewed measures. Finally, discussions on challenges and future research are provided.
\end{abstract}

Keywords: Segregation, Spatial, Geographical Information System (GIS), Quantitative Indices

\section{Introduction}

Segregation can be broadly defined as the degree of spatial separation between two or more population groups in a region (e.g., Newby, 1982). The uneven distribution of groups across urban space has long been a fundamental characteristic of modern cities (Lloyd et al., 2015; Wong, 2016). On the one hand, segregation reflects social inequalities, and tells us something about how these inequalities are changing, as current debates on the surburbanisation of poverty show. They also reflect wider factors, notably historic inequalities or patterns of land use, as well as past efforts in planning intervention. On the other hand, segregation is important for its influence on society and social relations. Many argue that it acts as a positive feedback mechanism, exacerbating inequalities not just reflecting them; this is the thrust of the neighbourhood effects literature (e.g. van Ham et al., 2012). Others see current levels of segregation as benign or even advantageous for society. No matter how segregation affects social welfare, 
better understating the degree and nature of segregation in a region is critical to effective policy making in relation to social equity (Johnston et al., 2014).

There has been a long history of efforts to derive measures which summarise segregation, reducing the complexity of spatial relations to a single indicator. Debate occurred around the different dimensions along which segregation could be conceptualised (Massey and Denton 1988) and around the principles on which measures should be selected (James and Taeuber, 1985). For example, Massey and Denton (1988) proposed five dimensions of segregation: evenness, exposure-isolation, concentration, centralization and clustering. It has also been argued, however, that they can be reduced to evenness-clustering and exposureisolation (Reardon and O'Sullivan, 2004), evenness-concentration and clustering-exposure (Brown and Chung, 2006), or the super-dimensions of separateness (summarizing (un)evenness, isolation, and clustering) and location (summarizing concentration and centralization) (Johnston et al., 2007). Apparently, the five dimensions overlap. Therefore, using them to classify or evaluate segregation measures may be confusing.

Further, the development of segregation measures has gone through several phases with different emphases. Sociologists and demographers led efforts initially. These measures evaluate the population distribution across areal units, population mix and potential interaction within areal units. The one most wellknown might be the dissimilarity index $D$ (Duncan and Duncan, 1955a), which can be formulated as in (1):

$$
D=\frac{1}{2} \sum_{i}\left|\frac{p_{i, g}}{p_{g}}-\frac{p_{i, \bar{g}}}{p_{\bar{g}}}\right|
$$

where $i$ is the index of spatial unit; $g, \bar{g}$ represent two population groups; $p_{g}, p_{\bar{g}}$ are total population of the two groups in the entire study region; $p_{i, g}, p_{i, \bar{g}}$ are population of groups $g, \bar{g}$ in spatial unit $i$, respectively.

Most of these measures like $D$ share a number of significant limitations. First, they throw away a great deal of spatial information, treating the urban system as being composed of discrete units, each divorced from surrounding areas as if it was an island. Second, they focus on a global summary for a city or region, assuming spatial relations are consistent across that place. Third, they look at residential space without paying attention to the varied locations in which people spend time across the course of a day.

A typical example is the "checkerboard problem" (White, 1983) that is often used to investigate the properties of segregation measures (Wong, 1999; Wong 
2002a; Dawkins, 2004; Harris, 2016), as shown in Figure 1. The four arrangements reflect different levels of segregation in most people's perceptions. However, the values of $D$ are the same, i.e. it fails to distinguish between different spatial arrangements. In fact, the value of $D$ will always be 1 as long as each spatial unit is occupied exclusively by one population group (White, 1983; Morrill, 1991; Wong, 1993).

Figure 1 about here

Recently, there have been varied attempts to overcome these limitations. Some researchers incorporated spatial elements into non-spatial indices to create the spatial versions of these measures (e.g., White, 1983; Morrill, 1991; Wong, 1993; Wong 2002a; Reardon and O'Sullivan, 2004; Wong, 2005). Others used inherently spatial measures, such as the centrographic measures, to summarize point locations (e.g., Wong, 1999; O'Sullivan and Wong, 2007). From a spatial perspective, segregated situations are often expected to have certain population groups highly clustered around certain areas. Spatial autocorrelation statistics have been used to evaluate the degree of similarity between neighbouring values and researchers have suggested using different spatial autocorrelation statistics to measure segregation (e.g., O'Sullivan and Wong, 2007).

All the above methods are bounded by the ecological approach and concerned about the residential pattern. Analysis is based on census or administrative units and data are aggregated population counts by groups. But the segregation notion refers to one's experience with other population groups, and the experience extends from residential space to other socioeconomic spaces. Thus, a new set of measures considering the activity patterns of individuals, and their interaction with other groups has been proposed (e.g., Wong and Shaw, 2011; Farber et al., 2015).

The aim of this paper is to review the development of spatial segregation measures following the four approaches described above. In addition, several related concepts relevant to implementing those indices, including representation of spatial interaction, spatial scales and statistical inference, are discussed. The motivation is both to enable researchers to make more informed choices between them but also to encourage their use in both academic and policy-related applications, so that we can achieve more sophisticated discussions about the meaning and importance of segregation.

This paper has two distinct contributions to existing literature. One is we discuss the significance testing of spatial segregation indices and present off-the-shelf software tools that can implement various spatial segregation measures, which 
has been ignored in previous reviews. The other is we discuss the challenges posed by two conceptual and methodological issues in the studies of segregation measures.

\section{Spatial Indices for Urban Segregation}

This section reviews spatial indices following the four approaches that characterize the development of spatial segregation measures, but is divided into five subsections. Efforts in spatializing traditional measures can be divided into two groups. Subsection 2.1 reviews the first group focusing on the family of dissimilarity indices often used in racial-ethnic segregation where the population is divided into discrete classes. Subsection 2.2 reviews the second group which belongs to the family of ordered indices focusing on income segregation or inequality. Subsection 2.3 reviews the second approach using spatial distribution indices to summarize population distributions. Subsection 2.4 discusses how spatial clustering statistics are used to reflect segregation. Spatial indices exploiting the activity space concept are reviewed in Subsection 2.5. Table 1 summarizes all the measures included in this review.

Table 1 about here

\subsection{Spatial Dissimilarity Indices for Discrete Population Groups}

Several extensions of $D$ explicitly incorporate spatial relationships between population groups in evaluating segregation levels. Examples include the work by White (1983), Morrill (1991), Wong (1993, 1998, 2005) and Reardon and O'Sullivan (2004).

Distance is a fundamental metric to reflect the spatial relationship between geographical features and people. The index of spatial proximity (SP) by White (1983) utilizes a distance function to represent how the potential for social interactions changes with distance. $S P$ can be viewed as an average of intragroup proximities, weighted by the proportions of each population group. It has a value of 1 if there is no difference in the distribution of population groups, while $S P>1$ indicates clustering of people of the same group whereas $S P<1$ implies members of one group live closer to another group. Grannis (2002) extended SP for the case of multiple population groups.

The distance metric can be reduced to adjacency, a topological concept that indicates spatial relationship. Adjacency has been used extensively to capture relationships between areal units in modeling spatial autocorrelation. Morrill (1991) incorporates a spatial adjacency term $w_{i j}$ in $D$ to represent the potential 
spatial interaction among population groups. The adjusted index $D$ (adj) is defined as:

$$
D(a d j)=D-\frac{\sum_{i} \sum_{j}\left|w_{i j}\left(z_{i, g}-z_{j, g}\right)\right|}{\sum_{i} \sum_{j} w_{i j}}
$$

where $z_{i, g}$ and $z_{j, g}$ are the proportions of group $g$ in spatial units $i$ and $j$, respectively; $w_{i j}$ has a value of 1 if units $i$ and $j$ are adjacent or 0 otherwise. The formulation of $D$ implies that populations in neighbouring units do not interact, as the boundary is treated as an absolute barrier of interaction. Given that most boundaries are not hard barriers to interaction, the second term in (2) was introduced to reflect the spatial interaction between adjacent units. If population shares between neighbouring units are different, interaction between these populations will lower segregation level. Subsequently, Wong (1993) proposed two modified versions of $D(a d j): D(w)$, which assumes longer shared boundaries between units enable more spatial interaction, and $D(s)$, which assumes the compactness of neighbouring units affects interaction between units.

The dissimilarity index $D$ and its spatial versions have a practical limitation: they compare only two groups while many societies have multiple racial-ethnic and socioeconomic groups. Morgan (1975) and Sakoda (1981) proposed a version for multiple groups, $D(m)$. Similar to $D, D(m)$ assumes no interaction among populations in neighboring units and is therefore aspatial. Wong (1998) proposed the spatial version, $S D(m)$, which has a structure identical to $D(m)$. What makes $S D(m)$ spatial is the use of the composite population counts instead of the original population counts. The composite population count of a unit includes the population in the reference unit and populations in neighboring units, removing the unit boundaries as the barriers to interaction. In other words, the concept of composite population counts assumes populations in neighboring units are mixed and should be counted toward the reference unit.

Wong (2005) argued that the composite population counts can replace the original population counts in $D$, and proposed a general index of spatial segregation, $G D$, which is a function of neighborhood size. Computing $G D$ over a range of neighbourhood sizes can depict how segregation varies with geographical scale (Wong, 2005).

The concept of composite population counts was adopted in several subsequent developments. Reardon and O'Sullivan (2004) developed a set of spatial segregation indices, including a spatial dissimilarity index $\widetilde{D}$ and a spatial information theory index $\widetilde{H}$, using a modified version of the composite population counts. Instead of treating the entire unit as part of the 
neighbourhood of a reference unit and counting its total population toward the reference unit's composite population, they proposed applying a spatial kernel centring at the reference unit. The kernel centre has a larger weight and farther locations have smaller weights. Thus, the modified composite population count of the reference unit is the weighted population counts of neighbouring units.

Instead of using the population density as in Reardon and O'Sullivan (2004), Feitosa et al. (2007) proposed a local generalized spatial dissimilarity (LGSD) index based on local population intensity. The local population intensity is a weighted average estimated by spatial kernel functions and independent of the size of spatial units. Further, by summing all the LGSD together, the generalized spatial dissimilarity index (GSD) can be obtained for the entire region.

\subsection{Income Segregation Indices for Ordered Groups}

A distinct feature of $D$ and its spatial extensions is that their application is limited to populations in discrete categories. Such indices are not suitable for continuous variables. To address this issue, a family of spatial ordering indices have been developed, largely built on the neighbourhood sorting index (NSI) and the Gini index.

NSI is an index of income segregation and is defined as the ratio of two standard deviations: one is of neighbourhood mean personal income and the other is of individual personal income, with larger values implying higher degrees of income segregation (Jargowsky, 1996). NSI is aspatial because it does not reflect how populations are segregated spatially by income, that is, shifting the populations and their incomes among areal units will not change the NSI value.

Jargowsky and Kim (2005) proposed a generalized NSI that accounts for the incomes in neighbouring spatial units. Based on the same concept of local population intensity as in GSD, Feitosa et al. (2007) extended NSI to a spatial NSI, which can be expressed as the ratio of the between-locality variance to the variance of the entire study area. Both generalized NSI and spatial NSI are smoothing techniques in the sense that the mean income of each neighbourhood is re-calculated by including its neighbours' average income, parallel to the concept of composite populations for count variables.

Reardon et al. (2006) argued that results of NSI-based measures are not often comparable as the incomes can change over time, but ranks of income are more robust. They proposed a spatial rank-order entropy index, $\widetilde{H}_{r}$, which only involves the rank ordering of incomes. $\widetilde{H}_{r}$ is a rank-order index, but is spatialized using $\widetilde{H}$, the spatial information index as mentioned above. $\widetilde{H}_{r}$ can also be 
calculated for each population group in order to compare the within-group income segregation (Reardon and Bischoff, 2011).

The Gini index, is widely used to measure (income) inequality (Duncan and Duncan, 1955a). Again, relocating people of different income levels will not change the index value because of its aspatial nature. Dawkins (2004) developed a standardised spatial Gini index $G_{S T}$ to measure racial segregation, which is calculated as the ratio of the spatial and aspatial Gini indices. In this case for racial segregation, both Gini indices are derived from the proportions between the two population groups, but for the aspatial index, the proportions are in a descending order and for the spatial index, the proportions are in a chosen spatial order. The measure indicates if the spatial arrangement of the population intensifies or dilutes segregation. Dawkins (2007) extended $G_{S T}$ to a spatial ordering index to measure spatial income segregation, where neighbourhoods are ranked spatially according to per capita income.

The concept of spatial order has also been employed in the centralization index (Duncan and Duncan, 1955b), which orders neighbourhoods using their distance to the city centre and thus represents the relative segregation to the centre of an urban area. Considering the polycentric nature of modern cities, Folch and Rey (2014) proposed a localised version of centralization index and argued that it could be an effective indicator of population segregation.

\subsection{Spatial Distribution Indices}

Another type of index focuses on the spatial distribution of the population. Since segregation implies spatial separation, segregation levels can be reflected by spatial correlation among population groups. Conceptually, if two population groups have similar spatial distributions, they are less separated and have low segregation. This type of measures only compares the spatial distributions among population groups and assumes that the locations of individuals are known or can be reasonably represented by certain point locations.

Wong (1999) suggested using centrographic measures such as the standard deviational ellipse to capture the spatial distribution of population groups. Comparing the standard deviational ellipses of different population groups can reflect their segregation level. Ebdon (1988) and Wong (1999) described how a standard deviational ellipse is fitted to a set of points, indicating the distribution of a population group. Thus, the segregation measure based on the ellipses can be expressed as follows (Wong, 1999):

$$
S=1-\frac{E_{1} \cap E_{2} \cap \ldots E_{m} \cap \ldots E_{M}}{E_{1} \cup E_{2} \cup \ldots E_{m} \cup \ldots E_{M}}
$$


where $E_{m}$ represents the ellipse of population group $m$ and $M$ is the total number of population groups. Conceptually, the index compares the spatial interaction and the union of the ellipses. The value of $S$ ranges from 0 (all groups have identical spatial distribution with ellipses overlapping each other perfectly) to 1 (groups have very different spatial distribution with no overlapping ellipses). Other measures capturing the spatial distributions of different populations can be compared to formulate other indices reflecting the segregation levels among population groups.

O'Sullivan and Wong (2007) considered not just where people are located, but also the intensity of their distributions. They overlaid the spatial kernel into every raster cell to estimate the population density. Population density levels of each population group over the region can be represented by a surface. Using these surfaces, they proposed a surface-based measure, which is defined in the same manner as $S$ in (3) but using volumes of the intersection and the union of probability density functions rather than ellipses.

\subsection{Spatial Clustering Indices}

As evenness-clustering is an important dimension of segregation, spatial clustering indices have been widely employed as an indicator of spatial segregation. Common examples include Location Quotient (LQ) (Isard, 1960), rule-based typology (Poulsen et al., 2001, 2002), Local Moran's I (Anselin, 1995), Getis-Ord local $G$ (Getis and Ord, 1992) and density-based measures (O'Sullivan and Wong, 2007).

$L Q$ was initially proposed to evaluate the concentration of an industry in a region in comparison to the national average. In terms of segregation, it is the ratio of a population group's share in a spatial unit to its share in the entire region (Brown and Chung, 2006). Poulsen et al. (2001, 2002) proposed a rule-based typology for classifying ethnic residential areas, accounting for both concentration and isolation. However, both of those approaches are virtually aspatial in the sense that they do not consider the spatial relationships among neighbouring units. Given that, Cromley and Hanink (2012) extended $L Q$ to the focal $L Q$ by incorporating the spatial structure of the reference population group using geographic weights.

According to Massey and Denton (1988) and others, concentration is a separate dimension in most conclusions. However, when an area has a concentration level higher than the others, how is that different from characterizing a clustering in that area? Conceptually, the five segregation dimensions may not be differentiable in certain situations. 
In spatial analysis, local indicator of spatial association (LISA) statistics, including Local Moran's I (Anselin, 1995) and the $G$ statistics (Getis and Ord, 1992) are usually employed to identify local clusters in spatial data. For example, in measuring racial segregation, the value of Local Moran's $I$ at spatial unit $i$ reflects how the proportion of a group in $i$ is similar to the proportions in neighbouring units. A high value of local Moran's I indicates a clustering of similar proportions (either high proportions reflecting a hot spot or low proportions reflecting a cold spot) and a low value indicates a clustering of dissimilar proportions. However, this application of spatial autocorrelation measure considers the degree of clustering of only one population group. Spatial relationships between groups are ignored.

Borrowing the approach of modeling spatial associations in $G$ statistics, Wong (2002a) developed a local index using the product of population counts between reference and neighbouring units, reflecting one group's exposure in the reference unit to another group in the neighbourhood. Meng et al. (2006) further extended Getis-Ord local $G$ by incorporating a social difference coefficient to handle ordinal social classes such as poor, middle and rich. These approaches are based upon spatial clustering indices, but are modified to measure exposure between population groups.

\subsection{Indices applied to Activity Space}

Most of the segregation measures in existing literature are intended for use with aggregated data to describe segregation patterns in residential space. Increasingly, the exclusive focus on the residential space has been challenged, as individual experiences in other socioeconomic spaces are also important to determine if one is segregated from other groups. Thus, locations in which people spend their daily lives, i.e. their activity spaces, may need to be considered. To reflect an individual's experience of segregation, the exposure dimension is considered the most effective (e.g., Schnell and Yoav; 2001; Wong and Shaw, 2011; Farber et al., 2012, 2015).

Activity space can be considered as "the subset of all locations within which an individual has direct contact as a result of his or her day-to-day activities"(Golledge and Stimson, 1997, p. 279). By measuring activity space from two dimensions (territorial and interactive), Schnell and Yoav (2001) developed socio-spatial isolation indices to represent an individual's isolation from others. Lower values of these indices represent higher levels of exposure to another group and larger values indicate higher tendencies of isolation from people of another group. 
Wong and Shaw (2011) extended the original exposure index (Lieberson, 1981) by replacing the areal units with individuals' activity spaces. Assume $\Omega_{i l}$ is the activity space of an individual $l$ of group $g$ in spatial unit $i$, the exposure of $l$ to group $\bar{g}$ within $\Omega_{i l}$ can be defined as:

$$
E_{i l, g \times \bar{g}}=\left(\frac{1}{p_{i l, g}}\right)\left(\frac{p_{i l, \bar{g}}}{t_{i l}}\right)
$$

where $p_{i l, g}$ and $p_{i l, \bar{g}}$ are the total population of groups $g$ and $\bar{g}$ within activity space $\Omega_{i l}$, respectively; $t_{i l}$ is the total population $l$ is exposed to within $\Omega_{i l}$. $E_{i l, g \times \bar{g}}$ in (4) can be extended to measure the exposure of all the people from group $g$ in unit $i$ to group $\bar{g}$ by utilizing the union of $\Omega_{i l}$ for all $l s$ (individuals) of group $g$ (Wong and Shaw, 2011). Thus, the spatial exposure measure constrained by the activity space concept for individuals can be expanded to deal with population groups.

The general notion of exposure through activity space has been extended further. Farber et al. (2012) proposed an exposure index with a similar form to Getis-Ord local $G$ utilizing trip lengths within activity spaces, intending to account for the general tendency that the intensity of interaction is inversely related to distance. The activity space of a person can be formally represented by a space-time prism, which defines the individual's spatiotemporal boundary given one's activity sites over time. Applying the exposure index concept in the space-time prism representation of activity space, Farber et al (2013) proposed that the intersections of individuals' prisms indicate the area of social interaction potential. The method can be extended to evaluate the spatiotemporal exposure between population groups (Farber et al. 2015).

\subsection{Summary}

We classified measures into categories partly according to their ability to capture different dimensions of segregation. If we try to match these indices to the five segregation dimensions, most indices for the first three types focus on the evenness dimension of segregation. Note that evenly distributed populations should not be misconstrued as the only condition for no segregation. In fact, these three types of index evaluate how (dis)similarly populations are distributed over space. The fourth type of measure focuses on the clustering dimension, but several scholars argue that clustering and evenness may be combined. The last type of index focuses on the exposure dimension, reflecting how different groups may interact over activity spaces. 
These methods can also be classified as global, local and individual according to the units of observations. Global measures such as $D($ adj $), D(w)$ and $D(s)$ are intended to summarize the overall segregation level across the entire study area, providing little information about the spatial variations in segregation across the study area, which might be of interest especially for large areas. Local indices such as $L G S D$, focal $L Q$ and Local Moran's I assess segregation for each spatial unit, reflecting the segregation levels at the neighbourhood or local scale. Finally, individuals' activity spaces can be used to generate the indices describing individuals' segregation experience, such as $E_{i l, g \times \bar{g}}$ in (4).

\section{Discussion}

As spatial segregation indices are geographic in nature, they are also subject to two fundamental problems facing any spatial analysis: representation of spatial interaction and spatial scales. The recent literature has been light in assessing the reliability (i.e. statistical significance) of those index values. It should be noted that there are numerous ways to represent spatial interaction, analyse spatial scale and derive statistical significance. The review here focuses on the selected methods employed by the segregation measures discussed above. Finally, in order to successfully implement various measures, software tools are needed for both researchers and practitioners. These issues are discussed below.

\subsection{Representation of Spatial Interaction}

A key concern in measuring spatial segregation is how to represent spatial interaction among population groups. Distance has long been used as a simple indicator of spatial separation or connection moderated by a distance decay effect. Therefore, spatial segregation indices have employed distance-based functions (e.g., SP). Also, distance is used in delineating the local population intensity in LGSD. Another common way to quantify spatial interaction is by examining the common boundaries of neighboring units. Populations in neighboring spatial units are modeled to interact and lower segregation. Neighboring relationship is often captured in spatial weights which are used in many spatial segregation indices (e.g., $D($ adj)). Obviously, the choice of spatial weights will affect the resultant values of segregation indices. Intersection between population groups is assumed if they share space. This is the essence of the set of spatial distribution and activity-space oriented indices (e.g., $S$ in (3)).

While assuming that interaction reduces segregation is conceptually sound and including interaction in measuring segregation is logical, the current conceptualization is crude and highly generalized. Thus implementing the concept involves a great deal of subjectivity. Specifically, no spatial segregation 
indices mentioned so far include actual interaction between population groups. The best we can say about these indices is that they capture the potential of interaction. All indices assume people interact over enumeration boundaries regardless of what the boundaries are. Some indices assume people interact if they are "close by" or "in the same location". In reality, the degree and frequency of interaction between groups is driven by many factors, of which proximity is only one. The architecture and layout of buildings, street layout, traffic and pedestrian flow, how long people remain stationary in a particular space, language and cultural barriers, communication technology and the nature of interaction - whether it business-related, social, planned, unplanned, whether people talk to each other in the same space or interact virtually via phone or social media - all affect the intensity, degree and frequency of interaction between groups. So we may want to be more modest about the claims that can be made about how well measures based largely on spatial properties can capture the true nature of interaction. In effect, these measures merely reflect how close or far people are apart spatially and to what extent they share similar locations. In a world where human interaction is increasingly done electronically and therefore aspatial, to what extent does it does matter if different population groups are spatially "separated"? We are not saying that space doesn't matter, but how do we gauge how much it matters and whether it's importance changing?

\subsection{Spatial Scale}

Like all spatial analysis techniques, spatial segregation indices are constrained by the geographical scale or level of spatial aggregation employed. That is, index values will differ when the same index is applied to data aggregated by different methods. This is known as the modifiable area unit problem (MAUP) (Openshaw, 1984), which refers to the variability in analytical results due to changes in the spatial resolution and different zoning schemes for which spatial data are tabulated. The former refers to different granularities of spatial representation, while the latter means different ways to partition a region with the same spatial resolution.

Using smaller spatial units (i.e. finer spatial resolutions) will almost always lead to larger segregation index values, aspatial and spatial alike (White, 1983; Wong et al., 1999; Krupka, 2007). If population is more clustered, spatial measures would be less affected by varying spatial scale than aspatial measures (Wong, 1997; Wong et al., 1999). Using simulated data, Rey and Folch (2011) examined the properties of four segregation indices $-D, D_{a d j}, N S I$ and the generalized $N S I$, 
and found $D$ and NSI are more sensitive to the region size than their spatial counterparts.

The MAUP is inherent in the use of spatially aggregated data. As long as segregation measures rely on data tabulated by areal units, the scale and zonal dependencies of analysis results are not going to disappear. Segregation indices developed within the activity space framework mostly rely on individual level data. Using individual level data is a potential direction to avoid the MAUP effects on segregation measurements, but segregation based upon individual experience needs to be re-conceptualized as some notions such as evenness or clustering for areal unit data may not be applicable.

\subsection{Statistical Inferences}

As discussed above, spatial segregation measures can be sensitive to the representation of spatial interaction and spatial scale. It is desirable to know the uncertainty associated with the derived indices. This issue was investigated to a certain degree soon after the dissimilarity index became popular (e.g., Cortese et al. 1976). Several studies have attempted to address this issue for non-spatial segregation indices using bootstrap tests (Brülhart and Traeger, 2005). However, Lee et al. (2015) found that standard bootstrapping approaches can perform very badly in the presence of spatial autocorrelation and the new method developed by Lee et al. (2015) performs much better, which has been applied to an centralisation index on the significance of the index difference across space or time (Kavanagh et al., 2016) and can in principle be applied to a wide range of spatial segregation measures.

For spatial segregation, a common concern is to test the obtained measures against randomness. Asymptotic theory and Monte Carlo simulation are two common methods employed for significance testing against randomness. The former has been widely applied particularly to spatial clustering indices. A similar method was used to test the significance of $G$-based exposure measures by Farber et al. (2012). In Monte Carlo simulation, population groups are randomly rearranged over space and segregation indices recalculated. If the randomization process is repeated a larger number of times (e.g. 999), a pseudo $p$-value can be obtained, representing the probability that the original spatial population distribution presents a higher level of segregation than other possible spatial arrangements of the same population composition (Anselin, 1995). Feitosa et al. (2007) used Monte Carlo simulation to verify the significance of the identified segregation patterns. 
However, the randomization approach to test the significance of segregation indices has its limitations. For instance, when original population counts are randomly re-assigned to different areal units, the process implicitly assumes that an areal unit can accommodate any population size, disregarding the area of the unit or other physical constraints. This assumption is unrealistic, and therefore not all randomized distributions are feasible. Effective and conceptually sounded testing approaches are still warranted. In addition, randomness is often regarded as the underlying distribution for no segregation. But if two groups are distributed across areas in the same manner, the definition for no segregation according to $D$, then higher non-random distributions may still have no segregation. Therefore, the concept of no segregation is still fuzzy.

\subsection{Software Tools}

Computing spatial segregation indices usually requires examining spatial relationships and implementing spatial analyses, which could be daunting tasks for researchers not familiar with GIS. As a result, applications of spatial segregation measures have been constrained by the availability of relevant software tools. Common software tools that provide functions for some of the aforementioned segregation measures are listed in Table 1.

Some tools developed in early eras were built upon popular commercial GIS software, utilizing the spatial data manipulation and analysis functions in those packages (Wong and Chong, 1998; Apparicio, 2000; Wong, 2002b, 2003). For example, the ArcView extension implemented several measures reviewed above like $D(s)$ and $D(w)$ (Wong, 2002b). However, the fast-paced software environment quickly made these tools obsolete.

In recent years, open GIS initiatives have promoted the development of many open source and free software tools, which have greatly encouraged the adoption and use of spatial segregation measures. The $R$ package "seg"* has implemented several spatial segregation indices such as $S P$ and $D$ (adj). Another $R$ package "spatialsegregation" $\dagger$ provides a function for the spatial information theory index $\widetilde{H}$ but primarily focuses on the segregation or mingling in multitype spatial point patterns, and therefore is not included in Table 1 . The “CARBayes" $¥ R$ package implemented the inference by Lee et al. (2015) for the index of dissimilarity and for the local and global relative centralisation index. The stand-alone software, Geo-Segregation Analyzer (Apparicio et al., 2014) is a

\footnotetext{
${ }^{*}$ https://cran.r-project.org/web/packages/seg/index.html + https://cran.r-project.org/web/packages/spatialsegregation/index.html

$\neq$ https://cran.r-project.org/web/packages/CARBayes/index.html
} 
multi-platform application that can calculate more than forty residential segregation indices. Particularly, the results of local measures can be mapped in the built-in viewer, enabling exploration of spatial variations in segregation. A limitation at present is that the outputs are text based and cannot be easily used as inputs for further analysis or visual summary.

The advancement in using the internet as the computational platform enables websites to provide computational and analytical services. Oasis, $§$ an online system that computes many aspatial and spatial segregation and inequality measures, was developed by leveraging the internet as a platform to deliver analytical functions and services to the public. The backend of the system is supported by $R$ procedures and functions. Using web-delivery approach broadens the access to these specialized tools.

\section{Challenges}

Many issues discussed above have been addressed in the literature to some extent, but they have not been resolved "satisfactorily". Some of these issues have been touched upon in a recent review (Wong, 2016). Two additional challenges, one conceptual and one operational, are discussed further here.

Wong (2016) argues that despite decades of research on measuring segregation, a consistent and generally agreeable definition of segregation has not yet been formulated. Definitions of segregation may be determined operationally, depending upon the measures adopted. Then using different measures will change the definition (e.g. using the dissimilarity index $D$ and isolation-exposure indices assume different definitions of segregation). The five dimensions of segregation (Massey and Denton 1988) have been used frequently as definitions conceptually, but recent debates show the five dimensions can be mingled together in different ways.

The bottom line is deciding which measure should be used as we are not short of measures of segregation but they capture different aspects of this complex, multi-faceted, and poorly-defined social construct. The issue is whether the measures really capture what we expect or intend them to explain. Measuring segregation is often the means, not the ends. The objective of measuring segregation is often to explore the relationships between some notion of segregation and certain outcomes or practices, for example, social actions such as discrimination or prejudice, which may lead to the creation of segregated neighbourhoods, or health outcomes such as a higher disease burdens for certain

\footnotetext{
${ }^{\S}$ https://oasis.irstea.fr/
} 
population sub-groups in a relatively segregated environment. Many studies have postulated possible relationships between segregation and various outcomes (e.g. Grady and McLafferty, 2007), and the roles of segregation may be causal, moderating or confounding. The challenge, given a specific outcome or action, is which facet of segregation is the most relevant and which measure is the best to capture that facet. For example, one might be concerned that the population of a "segregated" neighbourhood is not provided a reasonable level of access to healthcare facilities. Measuring segregation in this case should capture the low level of accessibility to those facilities, but using the dissimilarity measure $D$ will not be effective. Unfortunately, the complex and multifaceted relationships between segregation and various outcomes are still not well understood.

A trend in advancing segregation measurement is the move from ecological or place-based approaches using spatially aggregated data to individual or peoplebased approach using individual-level data to focus on individual's experience (e.g., Wong and Shaw, 2011). The ecological approach has to deal with the (scale effect of the) MAUP, and to choose the appropriate areal unit or often the census enumeration unit as the proxy of neighbourhood for the analysis. The scale effect in choosing neighbourhoods of different sizes on segregation measurement is well documented (e.g., Wong, et al. 1999; Reardon et al., 2009). Switching to a people-based approach and using individual-level data does not make the neighbourhood selection issue go away or simpler, but in fact makes the issue more complicated conceptually and operationally. Although the literature has logically suggested using activity pattern or activity space to define one's neighbourhood, such proposal is not without problems (e.g., Kwan, 2012). Technically, a "neighbourhood region" can be constructed based on one's activity locations. However, this neighbourhood region, regardless which method is used to construct it, will include areas or places that the individual just passes through without stopping. Should those unvisited locations be included in the "neighbourhood"? Another practical question is for how long the activity data should be collected in order to determine the "representative" activity pattern. If only locations (points) of activities are considered, should the activity space just be a set of points with no areal dimension? While some of these issues seem to be trivial, they are nonetheless difficult practical but fuzzy conceptual issues in measuring segregation.

\section{Final Remarks}

Quantitative indices of segregation are commonly employed in social sciences to represent the degrees of separation between social and population groups, 
particularly the isolation of more disadvantaged groups, and they can be powerful tools in assisting public policy formulation and decision-making. The review conducted in this paper focuses only on spatial segregation measures, i.e. those which attempt to capture the spatial arrangement of population and the spatial interactions between groups in one way or another. Essential here is how to represent spatial interactions between population groups, spatial units, or individuals. Robust indices with values insensitive to changes in spatial scale are needed, but remain elusive. Further, determining the statistical significance is important to indicate the reliability of the obtained segregation values. The emerging open-source and online software tools have greatly facilitated the implementation of those spatial measures. Recent shifts from place-based to people-based measures of segregation were a significant conceptual advancement accompanied by the increasing availability of individual-level mobility data. However, the introduction of these new approaches also brought along the conceptual issues of defining "neighborhood" appropriately. We hope this review provides insights into future extensions or improvements on existing measures, and encourages wider use of these powerful techniques.

\section{References}

Anselin, L. (1995). Local indicators of spatial association-LISA. Geographical analysis, 27(2), 93-115.

Apparicio, P. (2000). Les indices de ségrégation résidentielle: un outil intégré dans un système d'information géographique (Residential segregation indices: a tool integrated into a geographical information system). CyberGeo: European Journal of Geography, 134.

Apparicio, P., J. C. Martori, A. L. Pearson, E. Fournier \& D. Apparicio (2014). An open-source software for calculating indices of urban residential segregation. Social Science and Computer Review, 32(1): 117-128.

Brown, L. A., \& Chung, S. Y. (2006). Spatial segregation, segregation indices and the geographical perspective. Population, space and place, 12(2), 125-143.

Brülhart, M., \& Traeger, R. (2005). An account of geographic concentration patterns in Europe. Regional Science and Urban Economics, 35(6), 597624. 
Cortese, C. F., Falk, F., \& Cohen, J. K. (1976). Further considerations on the methodological analysis of segregation indices. American Sociological Review, 41(4), 630-637

Cromley, R. G., \& Hanink, D. M. (2012). Focal location quotients: specification and applications. Geographical analysis, 44(4), 398-410.

Dawkins, C. J. (2004). Measuring the spatial pattern of residential segregation. Urban Studies, 41(4), 833-851.

Dawkins, C. J. (2007). Space and the measurement of income segregation. Journal of Regional Science, 47(2), 255-272.

Duncan, O. D., \& Duncan, B. (1955a). A methodological analysis of segregation indexes. American sociological review, 20: 210-217.

Duncan, O. D., \& Duncan, B. (1955b). Residential distribution and occupational stratification. American Journal of Sociology, 60: 493-503.

Ebdon, D. (1988). Statistics in Geography. New York, NY: Basil Blackwell.

Farber, S., O'Kelly, M., Miller, H. J., \& Neutens, T. (2015). Measuring segregation using patterns of daily travel behavior: A social interaction based model of exposure. Journal of Transport Geography, 49, 26-38.

Farber, S., Neutens, T., Miller, H. J., \& Li, X. (2013). The social interaction potential of metropolitan regions: A time-geographic measurement approach using joint accessibility. Annals of the Association of American Geographers, 103(3), 483-504.

Farber, S., Páez, A., \& Morency, C. (2012). Activity spaces and the measurement of clustering and exposure: A case study of linguistic groups in Montreal. Environment and Planning A, 44(2), 315-332.

Feitosa, F. F., Camara, G., Monteiro, A. M. V., Koschitzki, T., \& Silva, M. P. (2007). Global and local spatial indices of urban segregation. International Journal of Geographical Information Science, 21(3), 299-323.

Folch, D. C., \& Rey, S. J. (2014). The centralization index: A measure of local spatial segregation. Papers in Regional Science. DOI: 10.1111/pirs.12145. 
Getis, A., \& Ord, J. K. (1992). The analysis of spatial association by use of distance statistics. Geographical analysis, 24(3), 189-206.

Golledge, R. G. and Stimson, R. J. (1997). Spatial behavior: A geographic perspective. Guilford Press, New York.

Grady, S. C. and McLafferty, S. (2007). Segregation, nativity, and health: reproductive health inequalities for immigrant and native-born black women in New York City. Urban Geography, 28(4), 377-397.

Grannis, R. (2002). Discussion: segregation indices and their functional inputs. Sociological Methodology, 32(1), 69-84.

Harris, R. (2016). Measuring segregation as a spatial optimisation problem, revisited: a case study of London, 1991-2011. International Journal of Geographical Information Science, 30(3), 474-493.

Isard, W. (1960). Methods of Regional Analysis. Cambridge: MIT Press.

James, D. R., \& Taeuber, K. E. (1985). Measures of segregation. Sociological Methodology, 1-32.

Jargowsky, P. A. (1996). Take the money and run: economic segregation in US metropolitan areas. American sociological review, 984-998.

Jargowsky, P. A., \& Kim, J. (2005). A measure of spatial segregation: The generalized neighborhood sorting index. Ann Arbor, MI: University of Michigan, National Poverty Center, Working Paper Series.

Johnston, R. J., Poulsen, M. \& Forrest, J. (2007). Ethnic and racial segregation in U.S. metropolitan areas, 1980-2000: The dimensions of segregation revisited. Urban Affairs Review, 42(4): 479 - 504.

Johnston, R. J., Poulsen, M. \& Forrest, J. (2014). Segregation matters, measurement matters. In C. L. Lloyd, I G. Shuttleworth and D. W. S. Wong (et al.) Social-Spatial Segregation. Policy Press, Bristol, UK., pp. 13-44.

Kavanagh, L., Lee, D. \& Pryce, G. (2016). Is poverty decentralising? Quantifying uncertainty in the decentralisation of urban poverty. Annals of the American Association of Geographers, (Forthcoming). 
Kwan, M.-P. (2012) The uncertain geographic context problem. Annals of the Association of American Geographers, 102(5): 958-968.

Krupka, D. J. (2007). Are big cities more segregated? Neighbourhood scale and the measurement of segregation. Urban Studies, 44(1), 187-197.

Lee, D., Minton, J., \& Pryce, G. (2015). Bayesian inference for the dissimilarity index in the presence of spatial autocorrelation. Spatial Statistics, 11, 8195.

Lieberson, S., Peach, C., Robinson, V., \& Smith, S. (1981). An asymmetrical approach to segregation. In: Peach, C., Robinson, V., and Smith, S. (eds). Ethnic segregation in cities. Croom Helm, London, pp 61-82.

Lloyd, C. D., Shuttleworth, I., \& Wong, D. W. (Eds.). (2015). Social-spatial segregation: concepts, processes and outcomes. Policy Press.

Massey, D. S., \& Denton, N. A. (1988). The dimensions of residential segregation. Social forces, 67(2), 281-315.

Meng, G., Hall, G. B., \& Roberts, S. (2006). Multi-group segregation indices for measuring ordinal classes. Computers, Environment and Urban Systems, 30(3), 275-299.

Morgan, B. S. (1975). The segregation of socio-economic groups in urban areas: a comparative analysis. Urban Studies, 12(1), 47-60.

Morrill, R. L. (1991). On the measure of geographical segregation. In Geography research forum (Vol. 11, No. 1, pp. 25-36).

Newby, R. G. (1982). Segregation, desegregation, and racial balance: status implications of these concepts. The Urban Review, 14(1), 17-24.

Openshaw, S. (1984). The Modifiable Areal Unit Problem. Geobooks, Norwich, England.

O'Sullivan, D., \& Wong, D. W. (2007). A Surface - Based Approach to Measuring Spatial Segregation. Geographical analysis, 39(2), 147-168.

Poulsen, M., Johnston, R., \& Forrest, J. (2001). Intraurban ethnic enclaves: introducing a knowledge-based classification method. Environment and planning A, 33(11), 2071-2082. 
Poulsen, M., Johnson, R., \& Forrest, J. (2002). Plural cities and ethnic enclaves: introducing a measurement procedure for comparative study. International Journal of Urban and Regional Research, 26(2), 229243.

Reardon, S. F., \& Bischoff, K. (2011). Income inequality and income segregation. American Journal of Sociology, 116(4), 1092-1153.

Reardon, S. F., Farrell, C. R., Matthews, S. A., O'Sullivan, D., Bischoff, K., \& Firebaugh, G. (2009). Race and space in the 1990s: Changes in the geographic scale of racial residential segregation, 1990-2000. Social Science Research, 38(1), 55-70.

Reardon, S. F., Firebaugh, G., O’Sullivan, D., \& Matthews, S. A. (2006). "Measures of Socioeconomic Segregation." Paper presented at the 29th General Conference of the International Association for Research in Income and Wealth, Joensuu, Finland, August 20-26.

Reardon, S. F., \& O'Sullivan, D. (2004). Measures of spatial segregation. Sociological methodology, 34(1), 121-162.

Rey, S. J., \& Folch, D. C. (2011). Impact of spatial effects on income segregation indices. Computers, Environment and Urban Systems, 35(6), 431-441.

Sakoda, J. M. (1981). A generalized index of dissimilarity. Demography, 18(2), 245-250.

Schnell, I., \& Yoav, B. (2001). The sociospatial isolation of agents in everyday life spaces as an aspect of segregation. Annals of the Association of American Geographers, 91(4), 622-636.

van Ham, M., Manley, D., Bailey, N., Simpson, L., and Maclennan, D. (2012), Neighbourhood effects research: new perspectives, Dordrecht: Springer.

White, M. J. (1983). The measurement of spatial segregation. American Journal of Sociology, 1008-1018.

White, M. J. (1986). Segregation and diversity measures in population distribution. Population index, 198-221.

Wong, D. W. (1993). Spatial indices of segregation. Urban studies, 30(3), 559-572. 
Wong, D. W. (1997). Spatial dependency of segregation indices. The Canadian Geographer, 41(2), 128-136.

Wong, D. W. (1998). Measuring multiethnic spatial segregation. Urban Geography, 19(1), 77-87.

Wong, D. W. (1999). Geostatistics as measures of spatial segregation. Urban Geography, 20(7), 635-647.

Wong, D. W. (2002a). Modeling local segregation: a spatial interaction approach. Geographical and Environmental Modelling, 6(1), 81-97.

Wong, D. W. (2002b). Spatial Measures of Segregation and GIS. Urban Geography, 23(1), 85-92.

Wong, D. W. (2003). Implementing spatial segregation measures in GIS. Computers, Environment and Urban Systems, 27(1), 53-70.

Wong, D. W. (2005). Formulating a general spatial segregation measure. The Professional Geographer, 57(2), 285-294.

Wong, D. W. (2016). From Aspatial to Spatial, from Global to Local and Individual: Are We on the Right Track to Spatialize Segregation Measures?. In Recapturing Space: New Middle-Range Theory in Spatial Demography (pp. 77-98). Springer International Publishing.

Wong, D. W., \& Chong, W. K. (1998). Using spatial segregation measures in GIS and statistical modeling packages. Urban Geography, 19(5), 477-485.

Wong, D. W., Lasus, H., \& Falk, R. F. (1999). Exploring the variability of segregation index D with scale and zonal systems: an analysis of thirty US cities. Environment and Planning A, 31(3), 507-522.

Wong, D. W., \& Shaw, S. L. (2011). Measuring segregation: an activity space approach. Journal of geographical systems, 13(2), 127-145. 
Figure 1. Examples of the "checkerboard" problem (four different spatial layouts of two population groups represented by black and white grid cells)

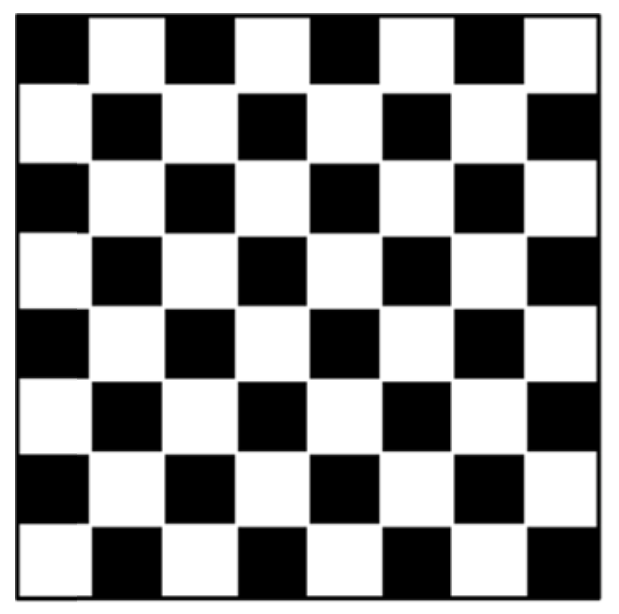

(a)

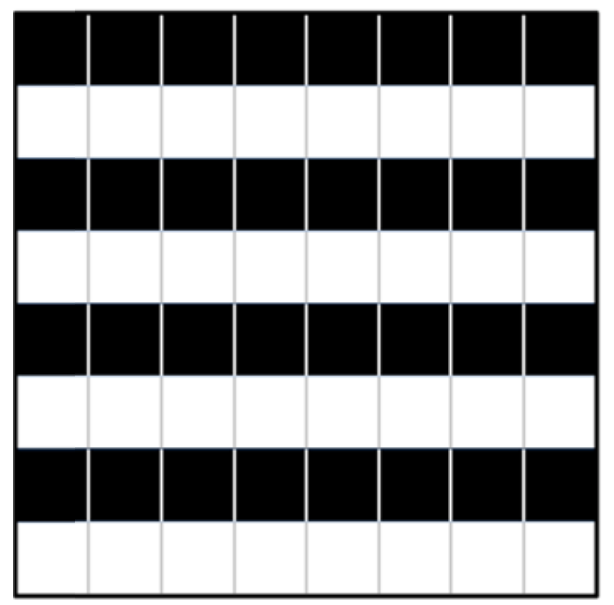

(c)

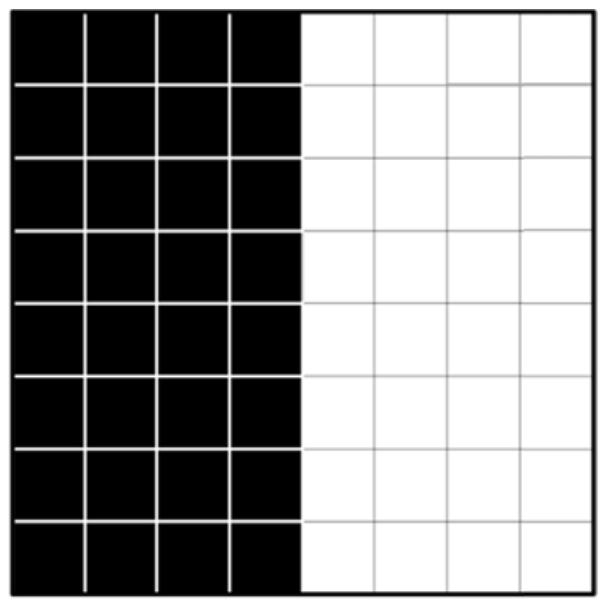

(b)

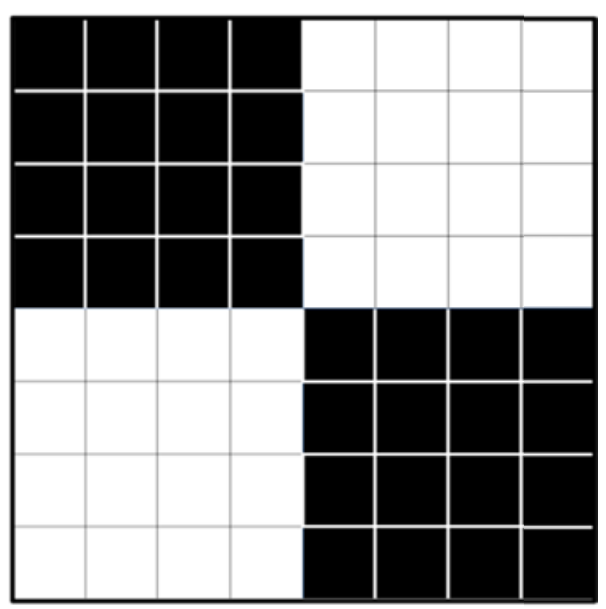

(d) 
Table 1 . Summary of segregation measures

\begin{tabular}{|c|c|c|c|c|c|c|}
\hline \multirow[b]{2}{*}{ Category } & \multirow[b]{2}{*}{ Description } & \multirow[b]{2}{*}{ Author } & \multicolumn{4}{|c|}{ Software } \\
\hline & & & $\begin{array}{l}\text { ArcView } \\
\text { extension }\end{array}$ & $\begin{array}{c}\text { "seg" } \\
(R \text { package })\end{array}$ & $\begin{array}{c}\text { Geo- } \\
\text { Segregation } \\
\text { Analyzer }\end{array}$ & Oasis \\
\hline \multirow{16}{*}{\begin{tabular}{l}
\multicolumn{1}{c}{ Spatial } \\
Spatial Dissimilarity \\
Indices for Discrete \\
Population Groups
\end{tabular}} & Dissimilarity index & Duncan and Duncan (1955a) & $\boldsymbol{v}$ & $\checkmark$ & $\boldsymbol{v}$ & $\checkmark$ \\
\hline & Gini index & Duncan and Duncan (1955a) & & & $\checkmark$ & \\
\hline & Centralization index & Duncan and Duncan (1955b) & & & $\checkmark$ & \\
\hline & Neighbourhood sorting index $(N S I)$ & Jargowsky (1996) & & & & \\
\hline & Typology for classifying ethnic residential areas & Poulsen et al. $(2001,2002)$ & & & $\boldsymbol{V}$ & \\
\hline & Location Quotient $(L Q)$ & Brown and Chung (2006) & & & $\checkmark$ & $\checkmark$ \\
\hline & & & & & & \\
\hline & Spatial proximity $(S P)$ index & White (1983) & & $\boldsymbol{\sim}$ & $\checkmark$ & \\
\hline & Multi-group $S P$ & Grannis (2002) & & & & \\
\hline & $\begin{array}{l}\text { Dssimilarity index incorporating common } \\
\text { boundary lengths and perimeter/area ratio }\end{array}$ & Wong (1993) & $\boldsymbol{v}$ & $\checkmark$ & $\checkmark$ & $\boldsymbol{v}$ \\
\hline & Spatial version of multigroup dissimilarity index & Wong (1998) & $\checkmark$ & & $\checkmark$ & $\checkmark$ \\
\hline & General index of spatial segregation & Wong (2005) & & & & \\
\hline & Spatial dissimilarity index & Reardon and O'Sullivan (2004) & & $\boldsymbol{\sim}$ & & \\
\hline & Spatial information theory index & Reardon and O'Sullivan (2004) & & $\checkmark$ & & \\
\hline & Generalized spatial dissimilarity (GSD) index & Feitosa et al. (2007) & & & & \\
\hline & Local GSD & Feitosa et al. (2007) & & & & \\
\hline
\end{tabular}




\begin{tabular}{|c|c|c|c|c|c|}
\hline \multirow{6}{*}{$\begin{array}{l}\text { Income Segregation } \\
\text { Indices for Ordered } \\
\text { Groups }\end{array}$} & Generalized NSI & Jargowsky and Kim (2005) & & \multirow{8}{*}{$\checkmark$} & \\
\hline & Spatial NSI & Feitosa et al. (2007) & & & \\
\hline & Spatial rank-order entropy index & Reardon et al. (2006) & & & \\
\hline & Standardised spatial Gini index $\left(G_{S T}\right)$ & Dawkins (2004) & & & \\
\hline & Spatial ordering index as an extension of $G_{S T}$ & Dawkins (2007) & & & \\
\hline & Localised version of centralization index & Folch and Rey (2014) & & & \\
\hline \multirow{2}{*}{$\begin{array}{l}\text { Spatial Distribution } \\
\text { Indices }\end{array}$} & Ellipse-based index & Wong (1999) & $\checkmark$ & & \\
\hline & Surface-based index & \multicolumn{2}{|l|}{ O'Sullivan and Wong (2007) } & & \\
\hline \multirow{5}{*}{$\begin{array}{l}\text { Spatial Clustering } \\
\text { Indices }\end{array}$} & focal $L Q$ & \multicolumn{2}{|l|}{ Cromley and Hanink (2012) } & & \\
\hline & Local Moran's I & Anselin (1995) & & & $\boldsymbol{V}$ \\
\hline & Getis-Ord local $G$ & \multicolumn{2}{|l|}{ Getis and Ord (1992) } & & \\
\hline & $\begin{array}{l}\text { Local segregation index incorporating spatial } \\
\text { interaction }\end{array}$ & Wong (2002a) & $\checkmark$ & & \\
\hline & $\begin{array}{l}\text { Extension of Getis-Ord local } G \text { incorporating } \\
\text { ordinal social classes }\end{array}$ & Meng et al. (2006) & & & \\
\hline \multirow{5}{*}{$\begin{array}{l}\text { Indices applied to } \\
\text { Activity Space }\end{array}$} & Socio-spatial isolation indices & \multicolumn{3}{|l|}{ Schnell and Yoav (2001) } & \\
\hline & $\begin{array}{l}\text { Exposure index using individuals' activity } \\
\text { spaces }\end{array}$ & \multicolumn{2}{|l|}{ Wong and Shaw (2011) } & & \\
\hline & Exposure index incorporating trip lengths & \multicolumn{2}{|l|}{ Farber et al. (2012) } & & \\
\hline & Index of social interaction potential & \multicolumn{2}{|l|}{ Farber et al. (2013) } & & \\
\hline & Exposure index using space-time prisms & \multicolumn{2}{|l|}{ Farber et al. (2015) } & & \\
\hline
\end{tabular}

